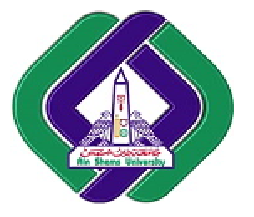

\title{
COMPLETE ENSEMBLE EMPIRICAL MODE DECOMPOSITION (CEEMD) FOR REAL-TIME SIGNAL DETRENDING IN IOT APPLICATIONS
}

\author{
M. T. Abduridha \\ A. S. Tolba \\ M. Z. Rashad \\ Computer Science Department,Faculty of Computers and Information, \\ Mansoura University, Egypt \\ Muayad.teto88@gmail.com \\ ast@astolba.com \\ Magdi_z2011@yahoo.com
}

\begin{abstract}
The Internet of Things (IOT) is a promising area which will boost the world economy. The constituent components of the IOT are smart objects which generate actuation signals or receive sensory signals which are usually noisy, have trend or has small signal-to-noise ratio. Processing these signals for filtering, detrending and enhancing the signal-to-noise ratio is crucial for embedding intelligence in these smart objects. This research discovers the potential of CEEMD in preparing signals for further intelligent applications such as event detection or pattern recognition in smart objects. Algorithms are presented for signal filtering, detrending and event detection based on a combination of both CEEMD, the autocorrelation function and the learning vector quantization classifier.The performance of the proposed algorithms is compared for both CEEMD and the least squares fit approach. The CEEMD has shown promising results.
\end{abstract}

Keywords: Internet of Things, Real-time Signal Detrending, Empirical Mode Decomposition, Complete Ensemble Empirical Mode Decomposition, Signal Denoising, Thresholding, Event Detection, Learning Vector Quantization.

\section{Introduction}

The weak and noisy signals produced by sensors impeded in smart objects of the IOT [15] and trend imposed on these signals as a result of the environmental changes are challenging the detection, prediction and event recognition algorithms which are essential part of smart objects. Trend is the low frequency part of a signal over which higher frequencies are carried and could be removed by filtering out the high frequency components from the signal.This research introduces a single algorithm for sensor signal denoising, detrending, and SNR enhancement in addition to extracting the signal discriminative features.In this paper a system that completely depends on CEEMD is implemented for real-time signal denoising, detrending, thresholding and event detection. Figure 1 shows the system layout. 


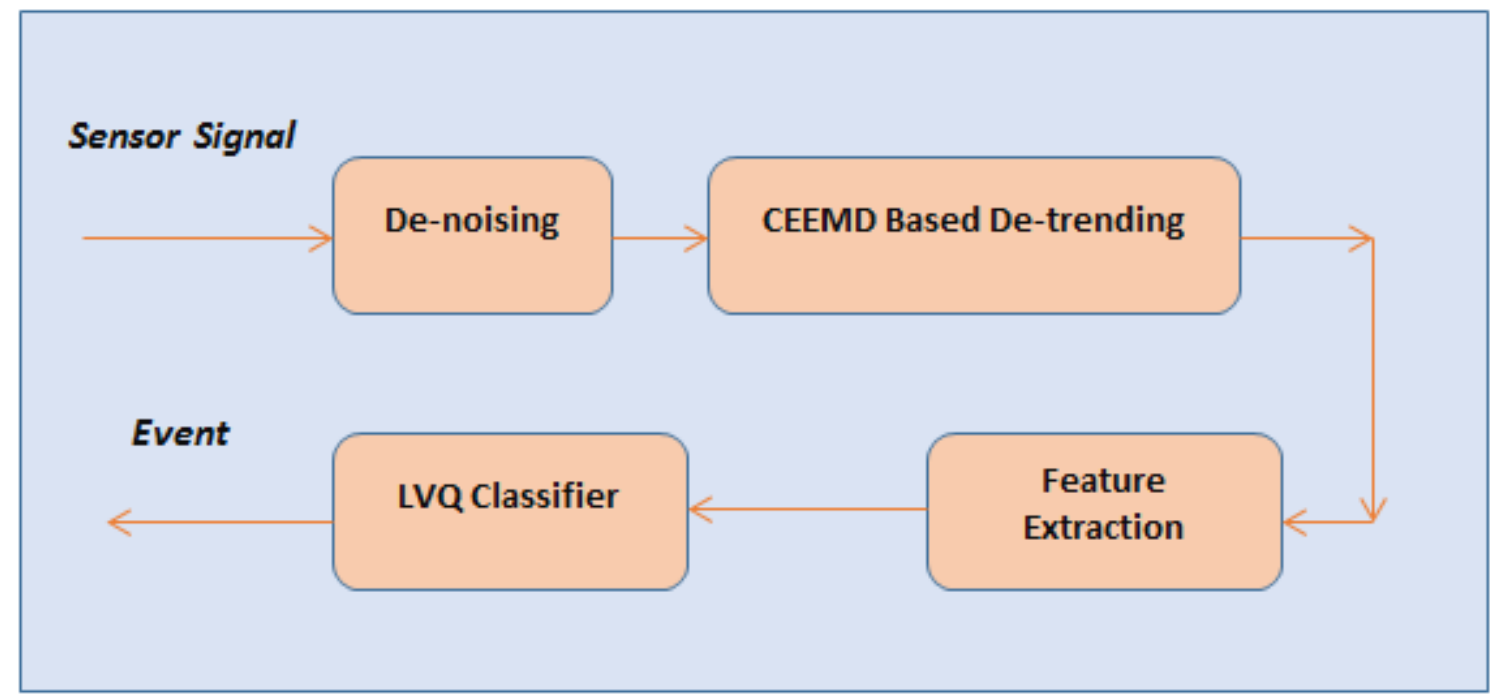

Figure 1: System Layout

\section{Empirical Mode Decomposition (EMD)}

The Empirical Mode Decomposition (EMD) is a recent signal decomposition approach which outperforms many signal processing approaches such as the Fourier Transform, Wavelet Transform. EMD decomposes a given signal into a set of Intrinsic Mode Functions (IMFs) using a sifting process according to the following algorithm [10-9]. An EMD algorithm decomposes adaptively the signal $x(t)$ into intrinsic mode functions $c_{i}(t), i=1,2, \ldots, n$ and into residue $r(t)$ :

$$
x(t)=\sum_{i=1}^{n} C_{i}(\mathrm{t})+r(t)
$$

wheren means the number of IMF functions. Residue $r(t)$ reflects the average trend of a signal $x(t)$ or a constant value.

1- Create upper envelope $E_{u}(t)$ by local maxima and lower envelope $E_{l}(t)$ by local minima of data $x(t)$.

2- Calculate the mean of upper and lower envelope.

$\mathrm{m}_{1}(\mathrm{t})=\mathrm{E}_{\mathrm{u} 1}(\mathrm{t})+\mathrm{E}_{\mathrm{i} 1}(\mathrm{t}) / 2$

3- Subtract the mean from original data

$\mathrm{h}_{1}(\mathrm{t})=\mathrm{x}(\mathrm{t})-\mathrm{m}_{1}(\mathrm{t})$

4- Verify that $h_{1}(t)$ satisfies conditions for IMFs. Repeat steps 1 to 4 with $h_{1}(t)$, until it is an IMF.

5- Get first IMF (after $k$ iterations)

$\mathrm{c}_{1}(\mathrm{t})=\mathrm{h}_{1(\mathrm{k}-1)}(\mathrm{t})-\mathrm{m}_{1 \mathrm{k}}(\mathrm{t})$

6- Calculate first residue

$r_{1}(t)=x(t)-c_{1}(t)$

7- Repeat whole algorithm with $r_{1}(t), r_{2}(t), \ldots$ until residue is monotonic function. 
8- Aftern iterations $x(t)$ is decomposed according to equation (1).

\section{Complete Ensemble Empirical Mode Decomposition (CEEMD)}

The major problems with EMD and end effects and mode mixing. The CEEMD version of EMD solves the two problems according to the following algorithm [17] by adding both negative and positive noise to the signal $\mathrm{x}(\mathrm{t})$ to be analyzed:

1- Select the number of EMDs used in the Complete Ensemble

2- Generate a Gaussian noise signal

3- Generate two signals by adding the sensor signal to the noise signal and subtracting the noise signal from the sensor signal

4- Apply the basic EMD to both signals separately to generate to separate sets of IMFs

5- Repeat steps 2 to 5 until the ensemble number of step 1 is reached

6- Calculate the ensemble means of the generate IMFs.

\section{CEEMD based signal detrending}

To remove trend from the sensor signal, all IMS are summed except the residue which represents the trend of the signal according to the following formula:

$$
y(t)=\sum_{i=1}^{N-1} I M F_{i}(6)
$$

Where $\mathrm{N}$ is the number of IMFs resulting from the decomposition of the sensor signal.

\section{CEEMD based signal denoising}

Denoising of sensor signals is essential for accurate event detection. CEEMD is also used for signal denoising in addition to signal detrending. Table 1 shows the superior performance of CEEMD [5] compared to other recent techniques such as EMD DFA [8] [1] and morphological transforms [16].The major drawback is the high computational cost. IT results in lower Mean Square Error (MSE), lower Mean Absolute Error (MAE), higher Signal-to-Noise Ratio (SNR) and Peak Signal-to-Noise Ratio (PSNR).

Table 1: Comparison between different Denoising Methods

\begin{tabular}{|c|c|c|c|}
\hline Method/Indicator & CEEMD & EMDDFA & Morphological Operators \\
\hline Time & $53.25 \mathrm{~s}$ & $3.448 \mathrm{~s}$ & $2.850 \mathrm{~s}$ \\
\hline MSE & $\mathbf{1 2 . 6 3 8}$ & 13.71 & 14.223 \\
\hline SNR & $\mathbf{1 1 . 8 1 4}$ & 11.46 & 11.30 \\
\hline PSNR & $\mathbf{2 3 . 3 0 3}$ & 22.95 & 22.79 \\
\hline Cross correlation & 0.9666 & 0.9626 & 0.9802 \\
\hline
\end{tabular}




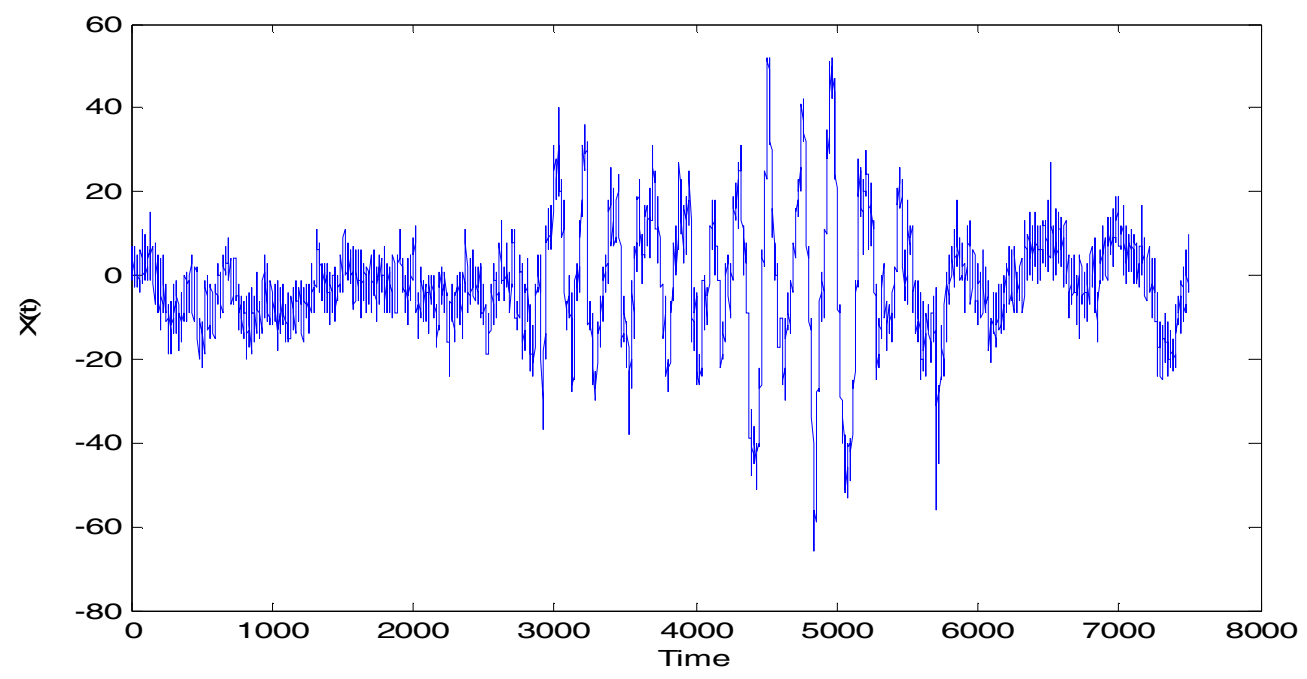

Figure 2: Simulated Noisy Signal

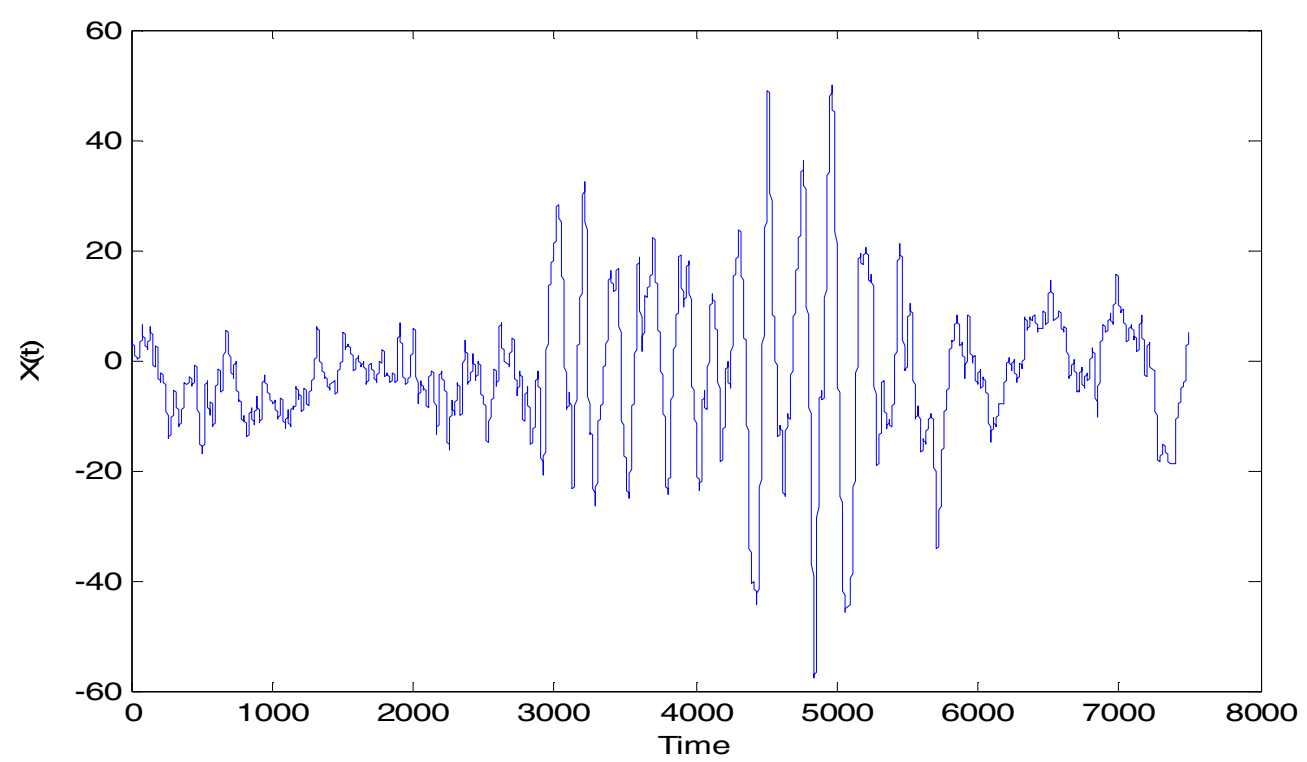

Figure 3: CEEMD Denoised Signal

\section{Experimental Set up and Results}

In this paper a real time sensory signal capture system is built around the Arduino Ethernet microcontroller, which is used to capture a real-time signal from a light sensor. An Arduino Ethernet board is connected to a light sensor. A micro USB cable is used to interface the Arduino Ethernet microcontroller with the PC. Some of wires and resistors are used for connecting the light sensor to the Arduino on a Breadboard. The light signal is degenerated by a light sensor (Light Detecting Resistor (LDR)), whose resistance decreases with increasing light intensity. The experimental set up is shown in figure (4). 


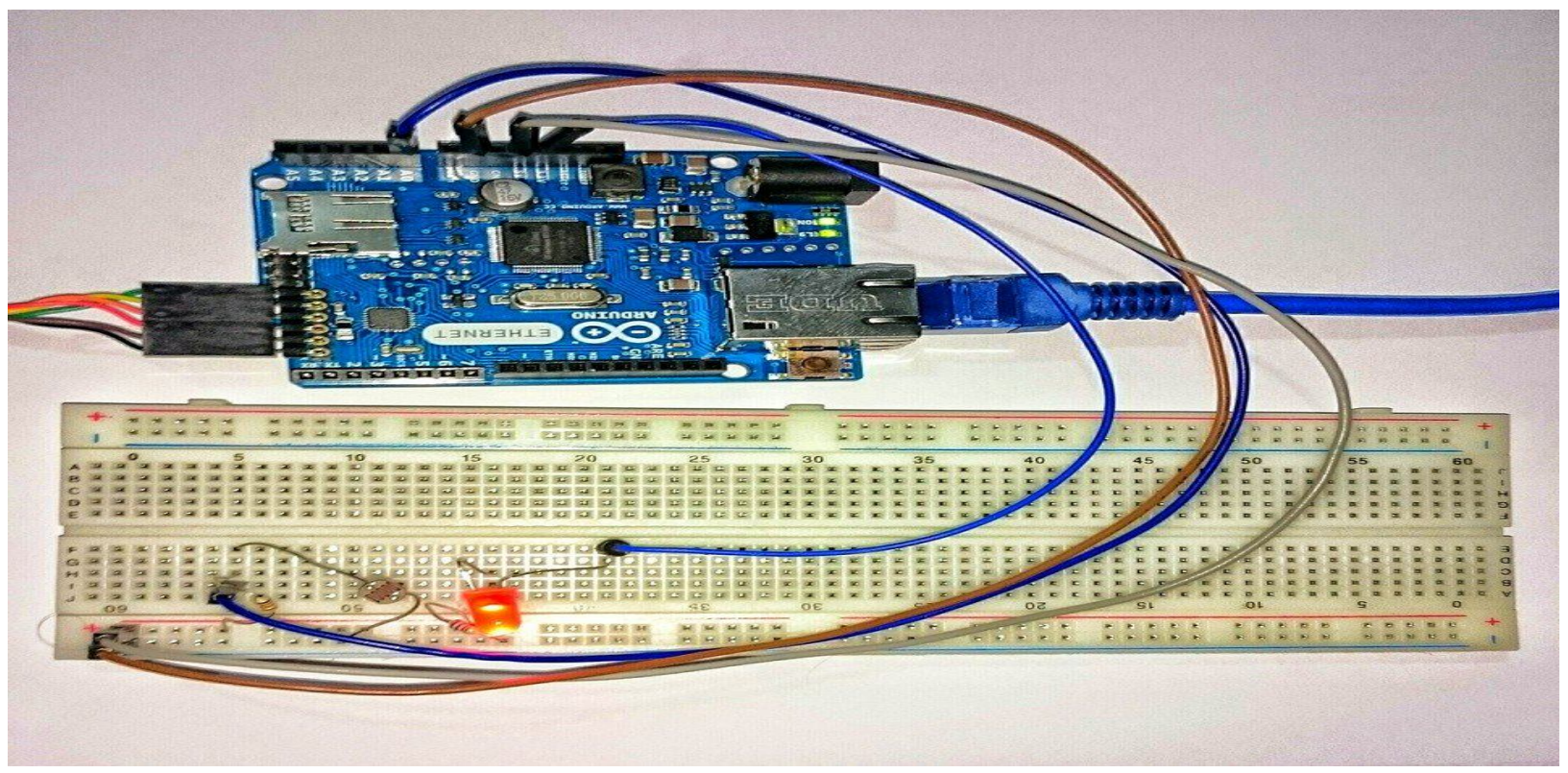

Figure.4. The Electronic Circuit

The control process is done through Arduino Ethernet [9-15] which contains a set of inputs (Analog Inputs) which can be used to read voltage (3.3-5V) restricted to the connected sensor. The sensor output signal captured in real time and then decomposed into a two sets of signals, the first set with high frequency and called (IMF) in the sense that it is changing quickly and the second set with low frequency that is, they are slowly changing, we set a low frequency signal so that we remove the trend (hard light located in the work environment), which is a straight line by referring volatile variable (light coming from outside the work environment). Using complete ensemble empirical mode decomposition algorithm we remove this signal and as a result we will get the trend free signal.

\section{Performance evaluation of the CEEMD based method}

To evaluate the performance of the proposed CEEMD algorithm, the Mean Square Error (MSE): $\mathrm{MSE}=\sum_{n=1}^{N}(\mathrm{Z}(\mathrm{t})-x(t))^{\wedge} 2$, and the Peak Signal-to-Noise Ratio (PSNR) via PSNR=MSE/ $\mathrm{x}(\mathrm{t})$ as are estimated.The table (2) compares the performance of the CEEMD with that of the well known least squares fit algorithm for a synthetic signal $\mathrm{X}(\mathrm{t})$.

Table 2 Performance comparison between the CEEMD and LSF detrending

\begin{tabular}{|c|c|c|}
\hline & CEEMD & LSF \\
\hline MSE & $1.2926 \mathrm{e}+005$ & $1.5436 \mathrm{e}+005$ \\
\hline PSNR & $7.068670 \mathrm{db}$ & $6.297807 \mathrm{db}$ \\
\hline SNR & $0.157091 \mathrm{db}$ & $0.021998 \mathrm{db}$ \\
\hline
\end{tabular}

\section{Feature Extraction and Classification for Sensory Event Detection}

To detect any abnormal event in a sensory signal (light or temperature), the detrended and denoised signal is used to extract a highly discriminative feature set which includes the following features: 
Skewness, Kurtosis and the sum of the ACF values for 10 lags are calculated according to the following formulas:

$$
\text { Kurtosis }=\frac{\mu^{4}}{\sigma^{4}}
$$

Where, $\mu^{4}$ is the fourth moment about the mean $\mu$ of the ACF values and $\sigma$ is the standard deviation of the ACF.

$$
\text { Skewness }=\frac{1}{N} \sum_{i=1}^{N}\left(\frac{x_{i}-\mu}{\sigma}\right)^{3}
$$

Where, $\mathrm{N}$ is the number of samples.

The Autocorrelation function $R(\tau)$ of the sensor signal $\mathrm{x}$ of $\mathrm{N}$ samples is calculated according to the following formula [14]:

$$
R(\tau)=\frac{1}{N-\tau} \sum_{i=0}^{N-\tau-1}(x(i) x(i+\tau))
$$

\section{Signal change event detection}

To classify the sensor signal as normal or abnormal a Learning Vector Quantization Neural Network Classifier [12] is trained on a large set of normal and abnormal signals. The training is performed on feature vectors having the three features extracted from the ACF of the sensor signal: skewness, kurtosis and sum of ACF values (Table 3).

Table 3 Feature sets for Normal and Abnormal Light Signals

\begin{tabular}{|c|c|c|}
\hline Feature / Status & Normal & Abnormal \\
\hline Skewness of ACF & 2.7085 & 0.3264 \\
\hline Kurtosis of ACF & 8.6386 & 1.6439 \\
\hline Sum of the ACF values at 10 lags & 0.4471 & 2.1903 \\
\hline Signal Status & Abnormal state & Normal state \\
\hline
\end{tabular}


The training set used by LVQ for detection of abnormal light change is extracted in real time as follows:

$\begin{array}{llcl}\text { Skewness } & \text { Kurtosis } & \text { Sum of ACF } & \text { Target Class } \\ 2.5201 & 8.0281 & 1.0071 & 1 \\ 2.6390 & 8.4025 & 0.6441 & 1 \\ 2.7406 & 8.7436 & 0.6018 & 1 \\ 2.6942 & 8.6035 & 0.7762 & 1 \\ -0.2253 & 1.7490 & 9.8001 & 2 \\ -0.2296 & 1.7667 & 8.8142 & 2 \\ 0.1094 & 1.5752 & 3.9537 & 2 \\ -0.1639 & 1.7462 & 9.5102 & 2\end{array}$

Where class 1 represents a normal light signal and class 2 represented a changed light signal due to an external interference.

\section{Real-Time Experimental Results and Discussion}

\section{Experiment 1: Normal Light State}

Figure 5-6, show a real-time light signal and its intrinsic mode functions. Figures 7 shows the results of signal detrending using both the CEEMD and LSF algorithms. The ACF of the detrended signal is shown in figure 8.

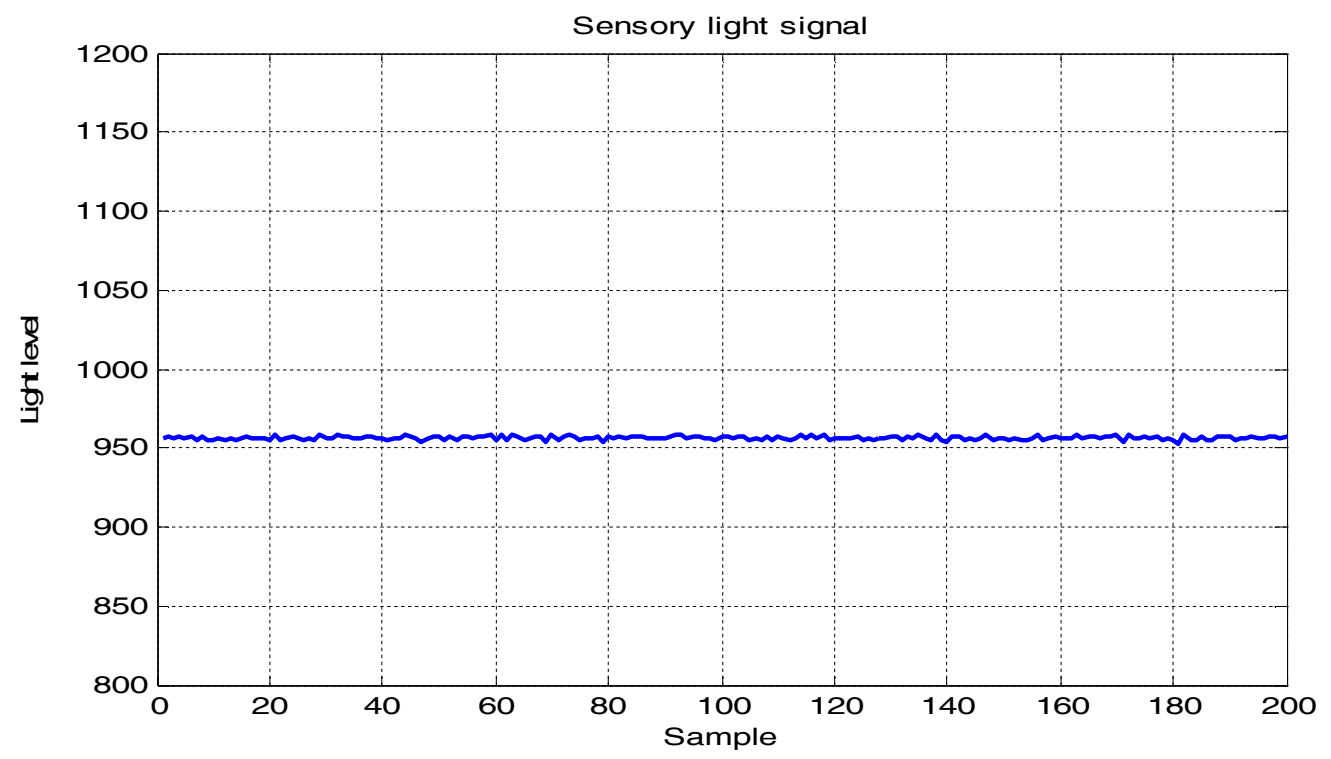

Figure 5: Normal Light Signal 


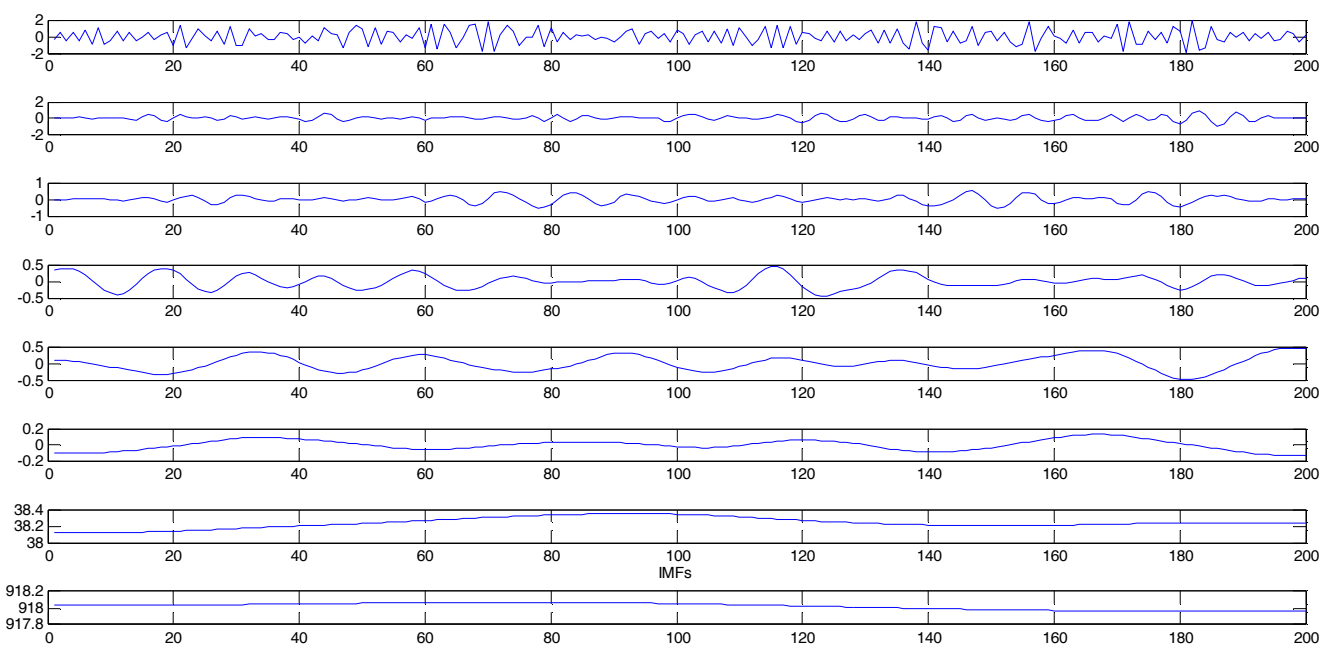

Figure 6: IMFs of the normal light signal
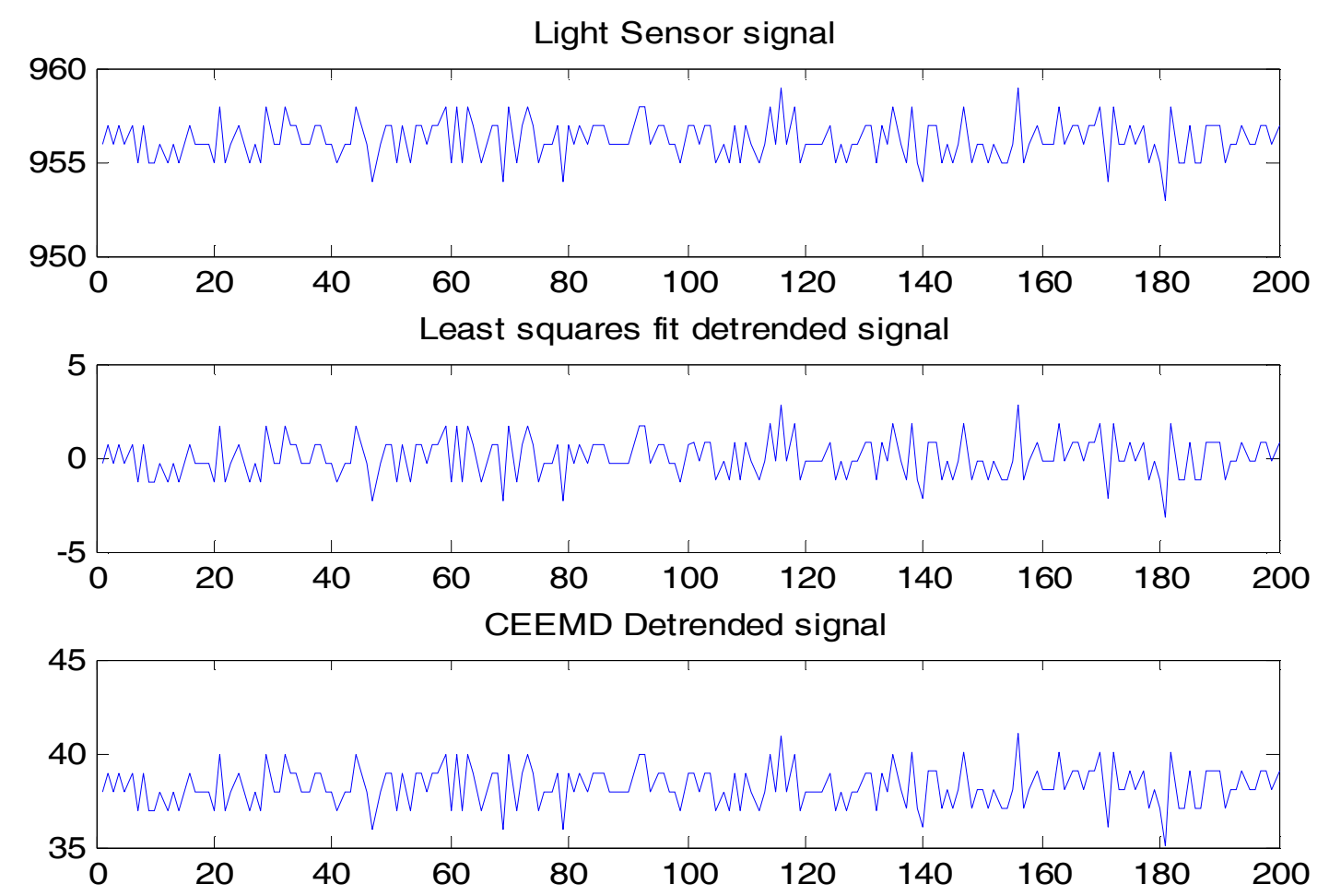

Figure 7: Detrending by both LSF and CEEMD methods 


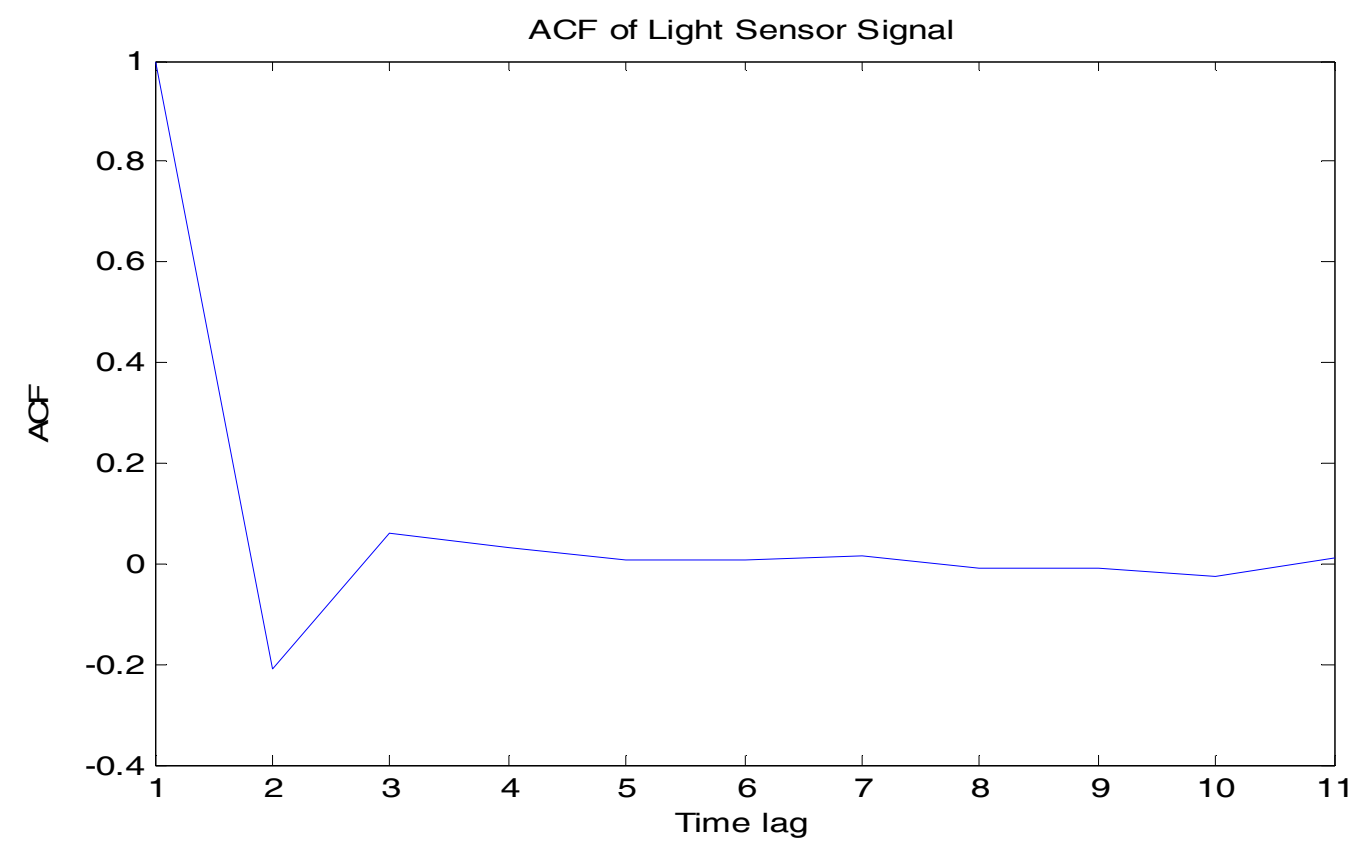

Figure 8: ACF of the detrended signal

\section{Performance comparison of both LSF and CEEMD:}

Table 5 Performance comparison between the CEEMD and LSF detrending of a Normal Temperature Signal

\begin{tabular}{|c|c|c|}
\hline & CEEMD & LSF \\
\hline Time & 10.203125 seconds & 0.109375 seconds \\
\hline MSE & 842785.752087 & 914470.582030 \\
\hline SNR & $0.354377 \mathrm{db}$ & $0.000005 \mathrm{db}$ \\
\hline PSNR & $0.379200 \mathrm{db}$ & \multirow{2}{*}{$0.024675 \mathrm{db}$} \\
\hline Skewness of ACF & 2.562262 & \\
\hline Kurtosis of ACF & 8.286071 & \\
\cline { 1 - 2 } Sum of the ACF values at 10 lags & 0.879909 & \\
\cline { 1 - 2 } Signal Status/LVQ class & Normal state & \\
\hline
\end{tabular}

\section{Experiment 2: Abnormal Light State}




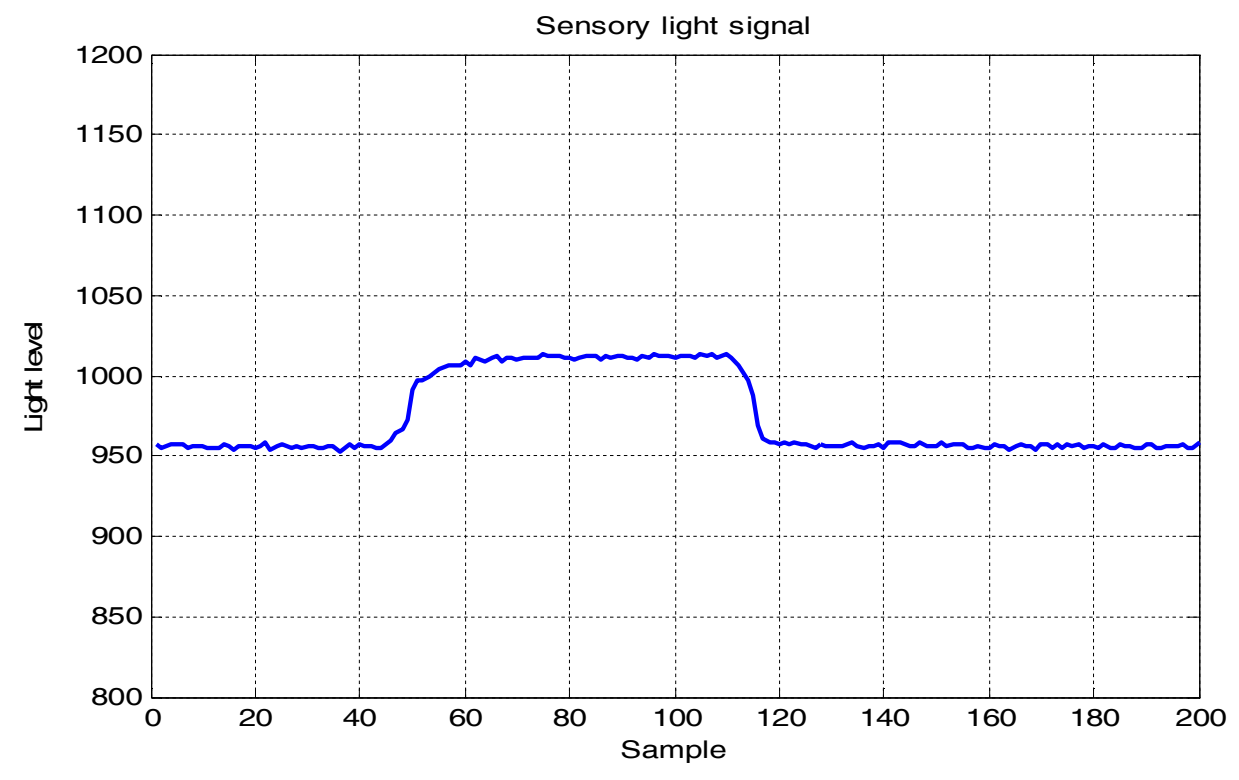

Figure 9: Abnormal light signal

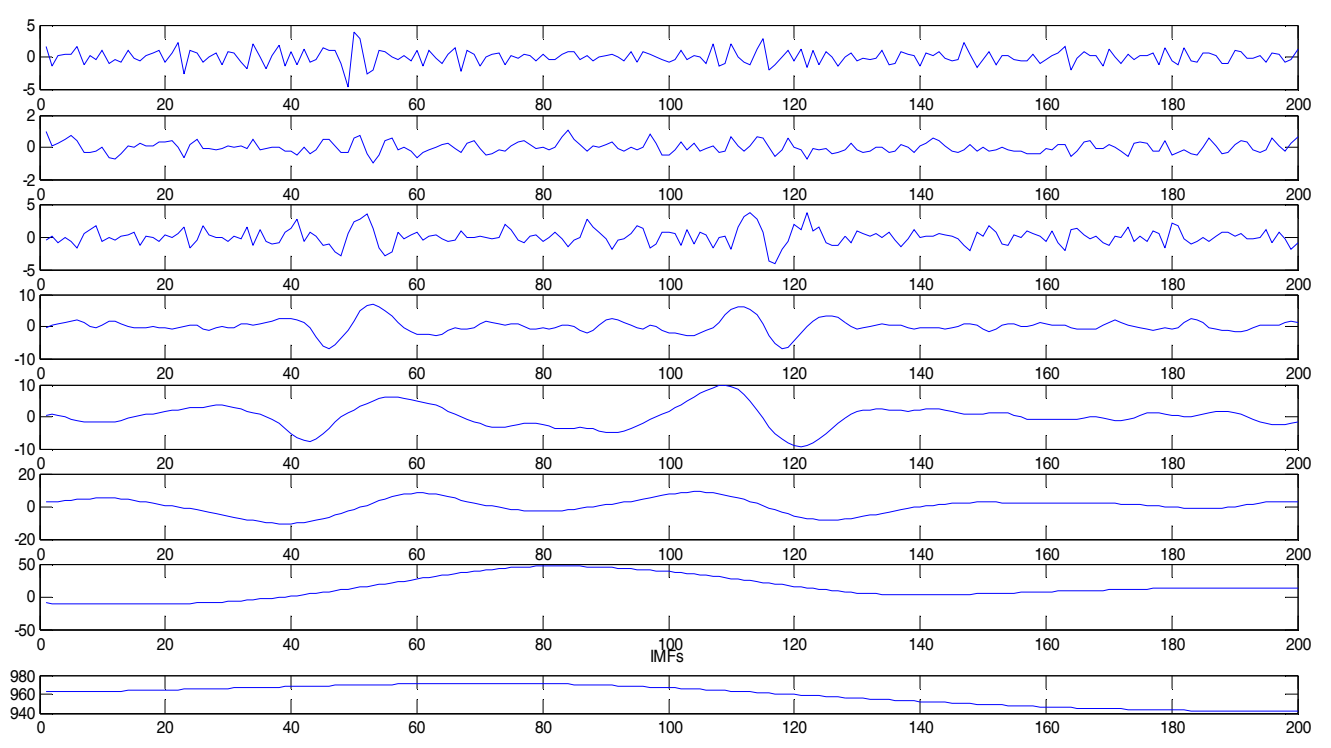

Figure 10: IMFs of an abnormal light signal 

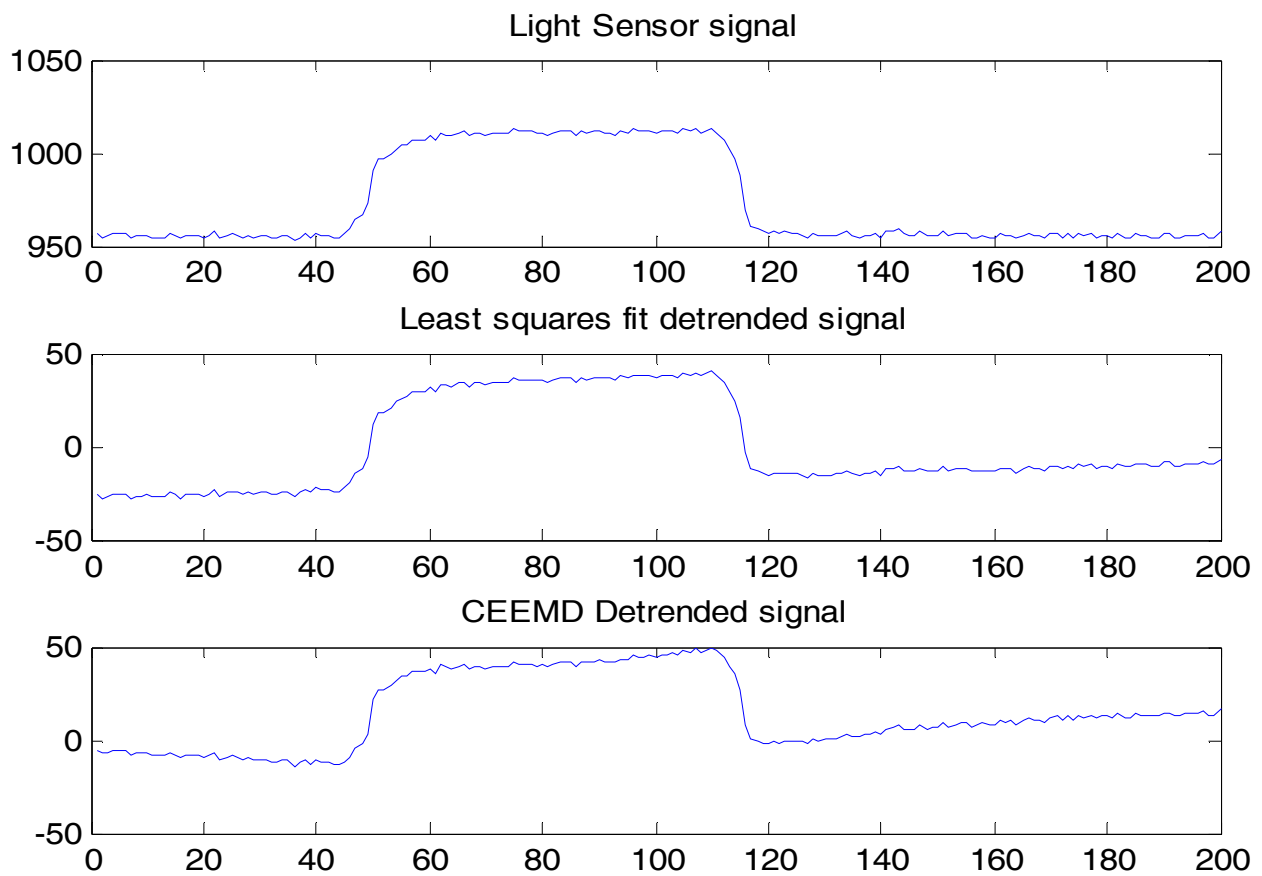

Figure 11: Dtrended signal by both LSF and CEEMD

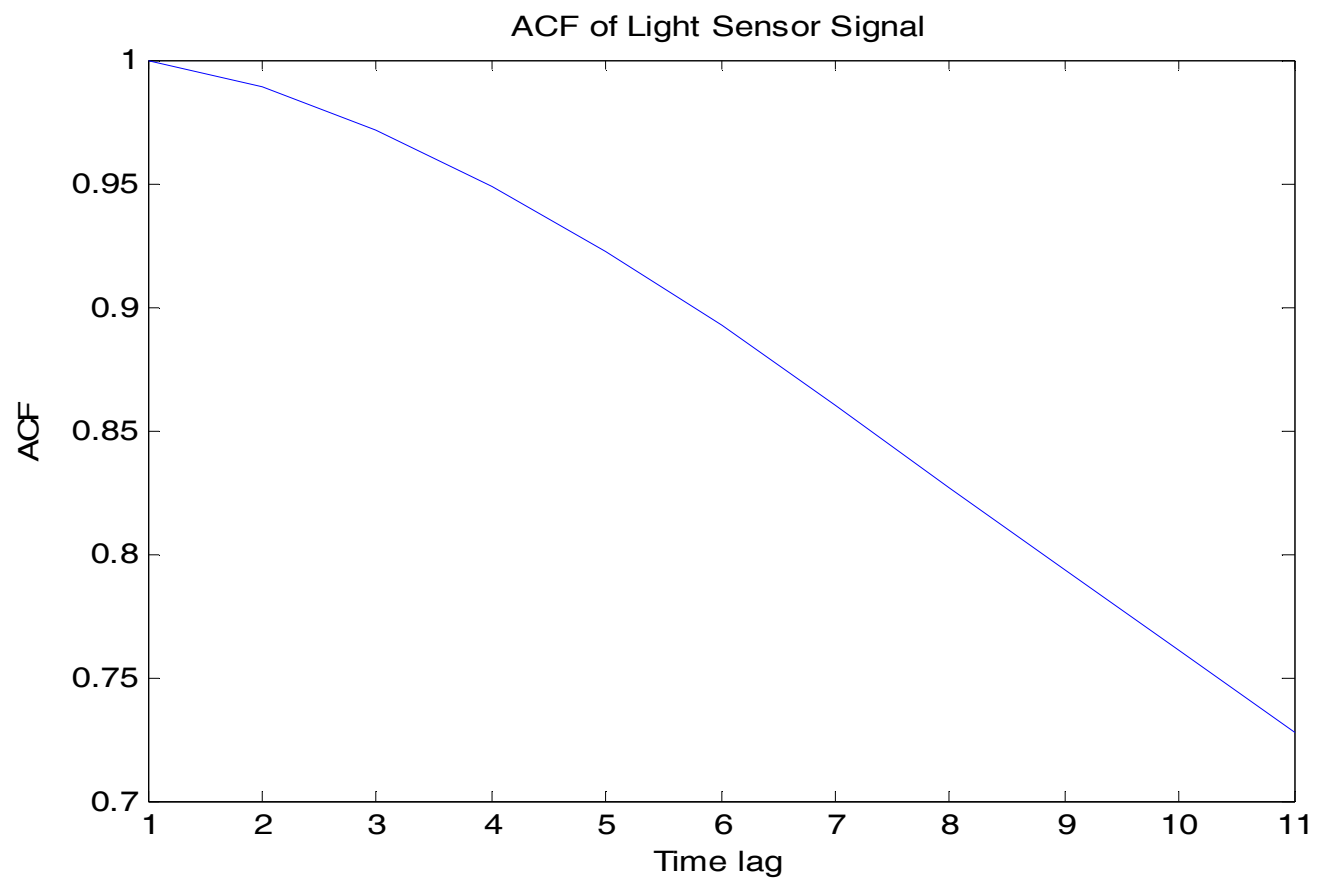

Figure 12: ACF of the detrended signal

\section{Performance Evaluation of both LSF and CEEMD:}

Table 6 Performance comparison between the CEEMD and LSF detrending of an Abnormal Temperature Signal 


\begin{tabular}{|c|c|c|}
\hline & CEEMD & LSF \\
\hline Time & 10.281250 seconds & 0.031250 seconds \\
\hline MSE & 926294.848920 & 966443.351835 \\
\hline SNR & $0.132751 \mathrm{db}$ & $0.002717 \mathrm{db}$ \\
\hline PSNR & $0.444696 \mathrm{db}$ & \multirow{2}{*}{$0.260425 \mathrm{db}$} \\
\hline Skewness of ACF & 0.269408 & \\
\hline Kurtosis of ACF & 1.733650 & \\
\hline Sum of the ACF values at 10 lags & 9.692990 & \\
\hline Signal Status / LVQ class & Abnormal state & \\
\hline
\end{tabular}

\section{Experiment 3: Abnormal Light State}

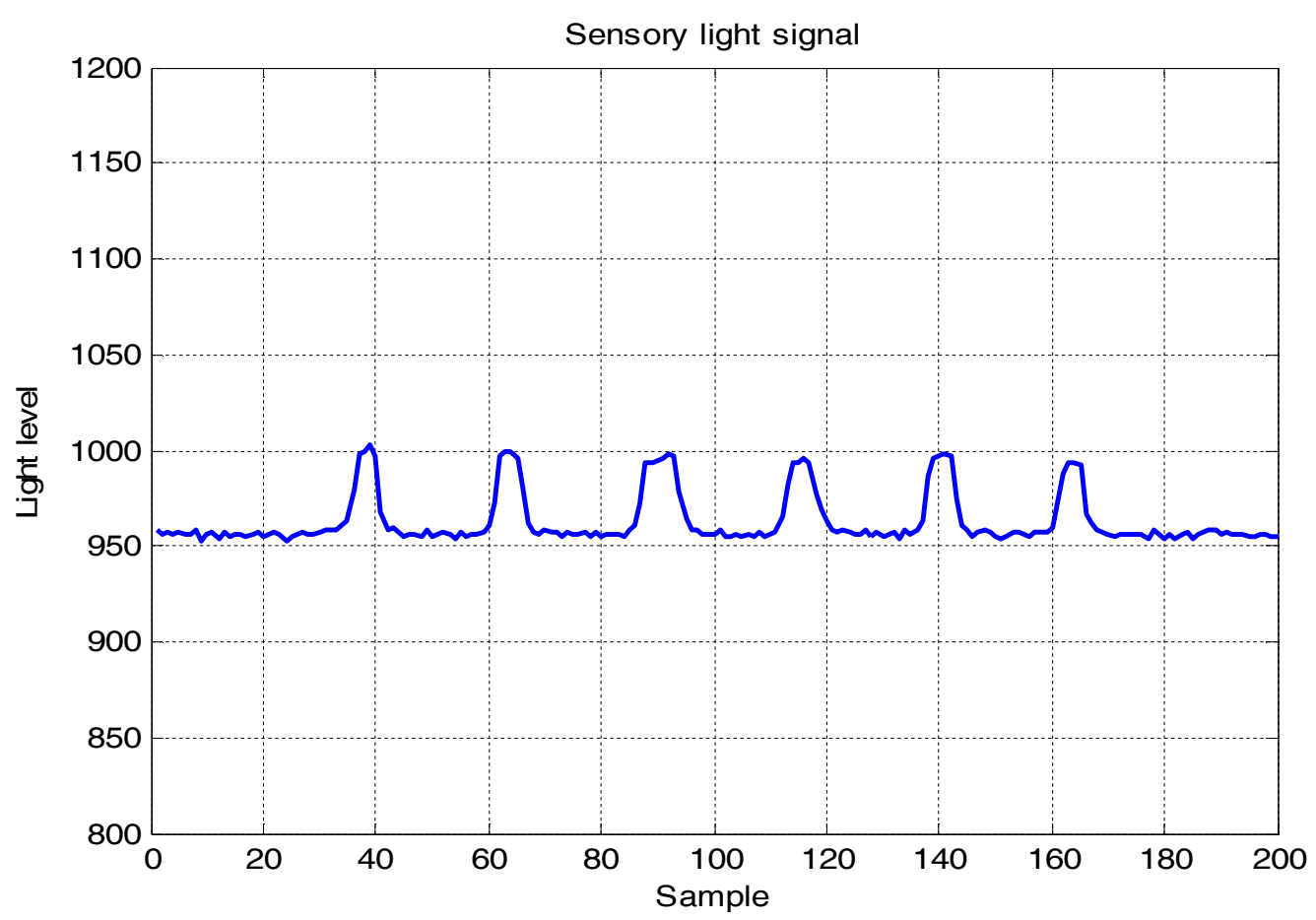

Figure 13: Abnormal light signal 

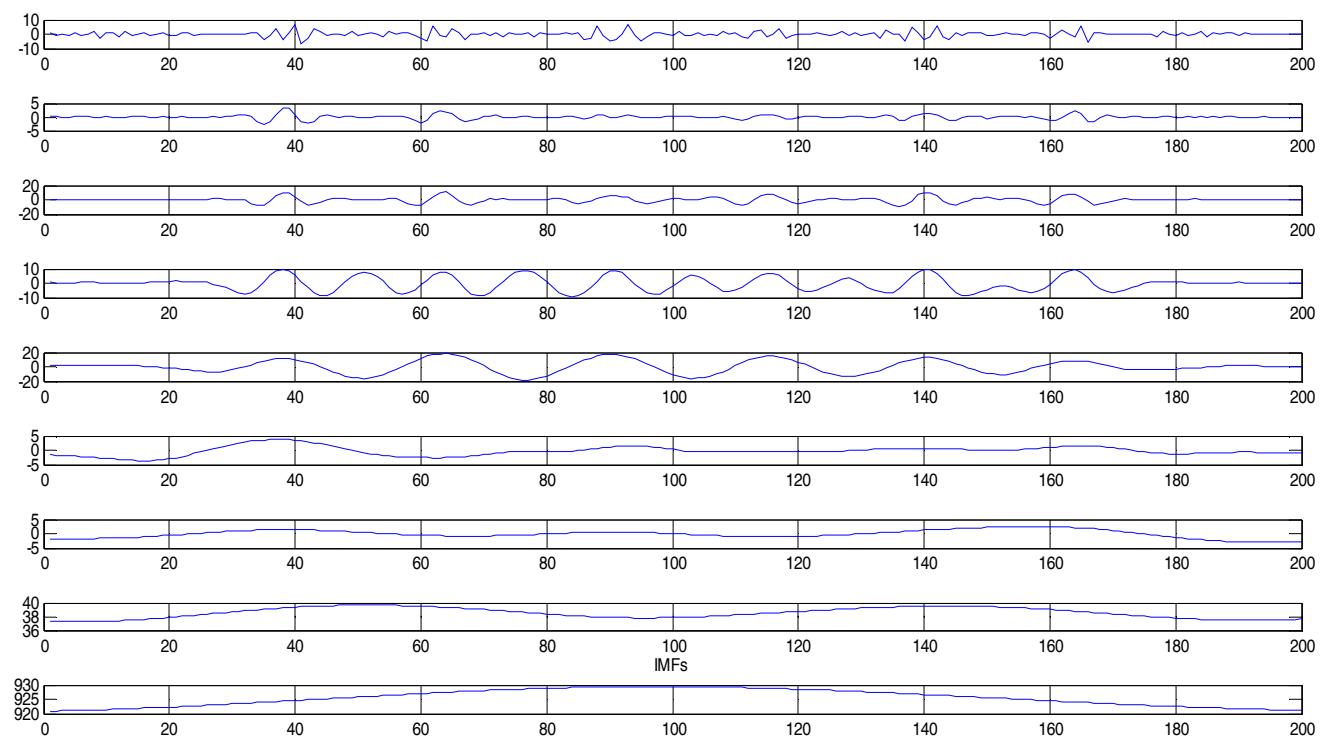

Figure 14: IMFs of an abnormal light signal
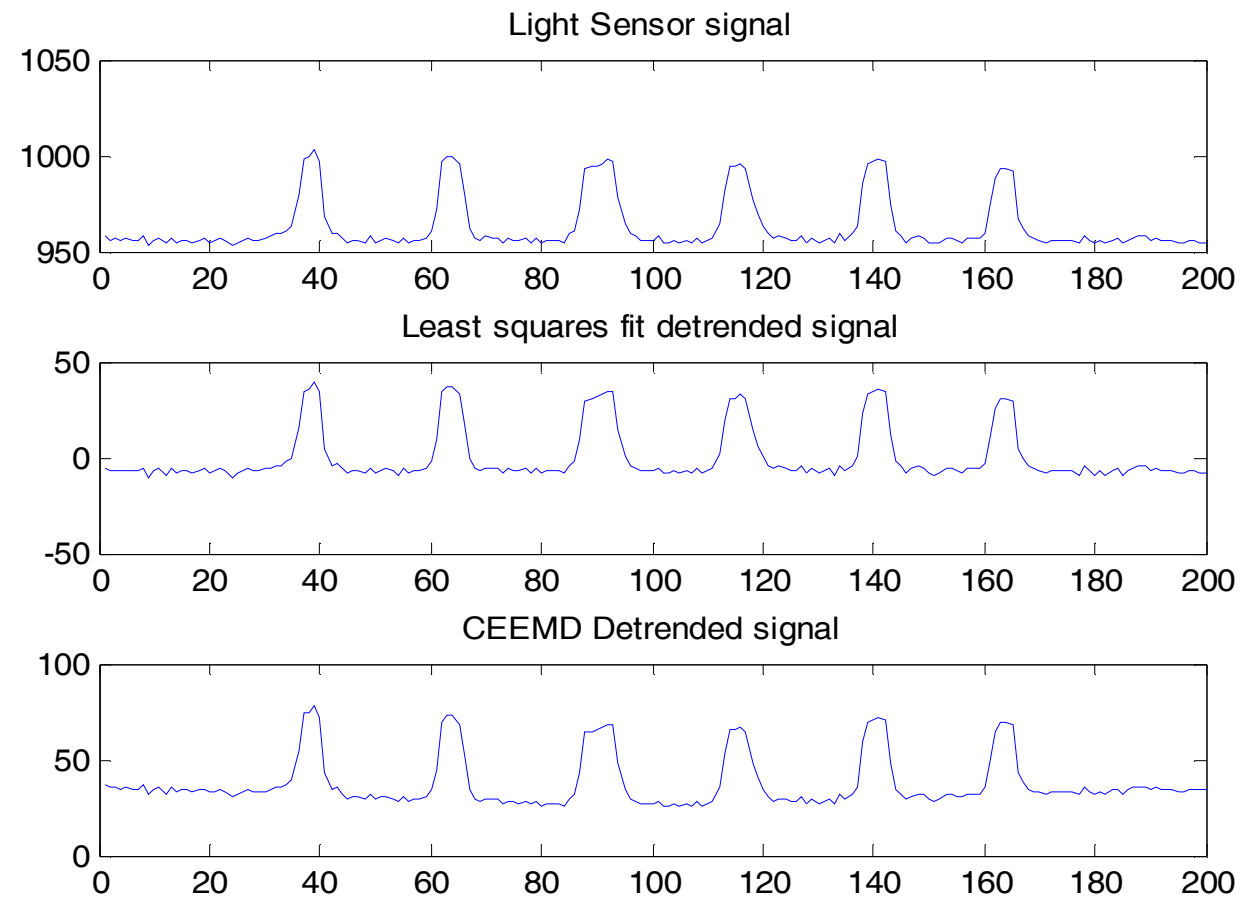

Figure 15: Dtrended signal by both LSF and CEEMD 


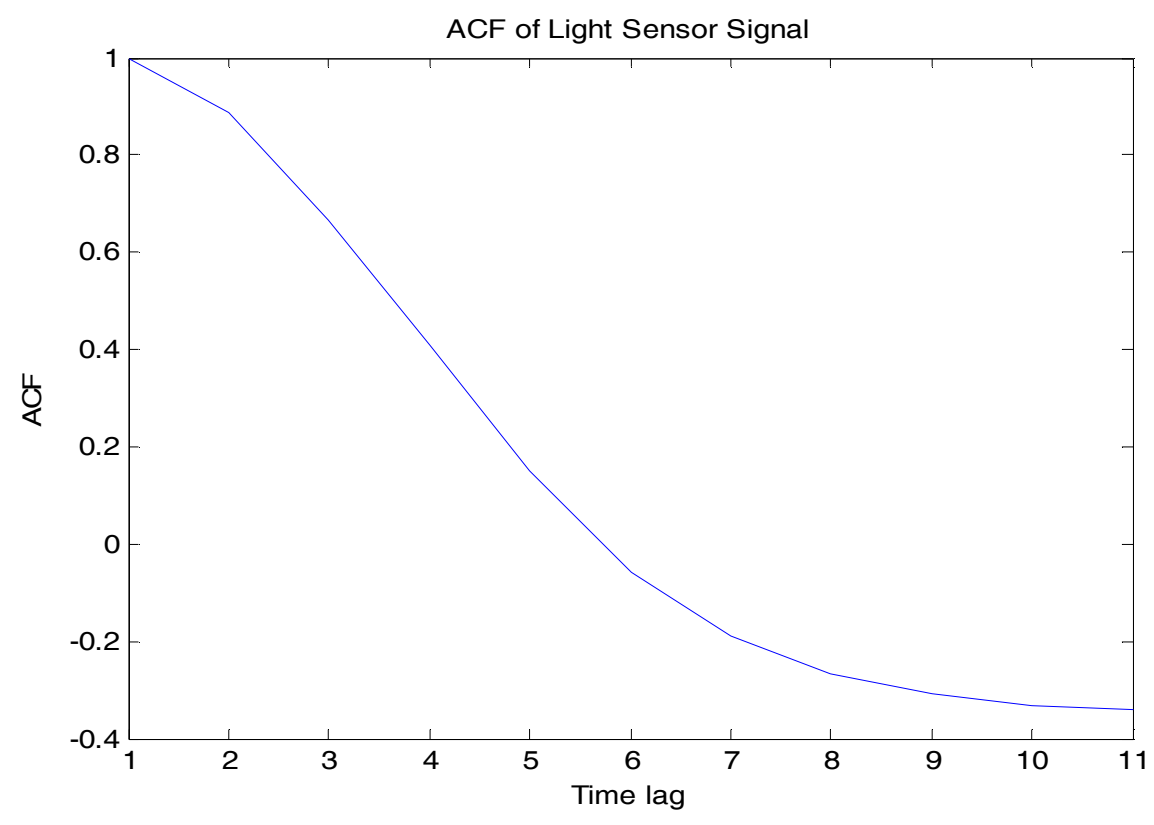

Figure 16: ACF of the CEEMD detrended signal

Tables 5-6 show that the CEEMD detrending approach results in better performance than thee LSF approach.

\section{Experiment 4: Simplified Signal Thresholding Based on Detrending}

To detect events in a cluttered signal or in a signal with a trend it seems impossible without removing the trend. This section shows how trend removal could simplify the thresholding of a given signal. Figure 17 shows a pulse overlaid on an exponentially decaying trend signal.

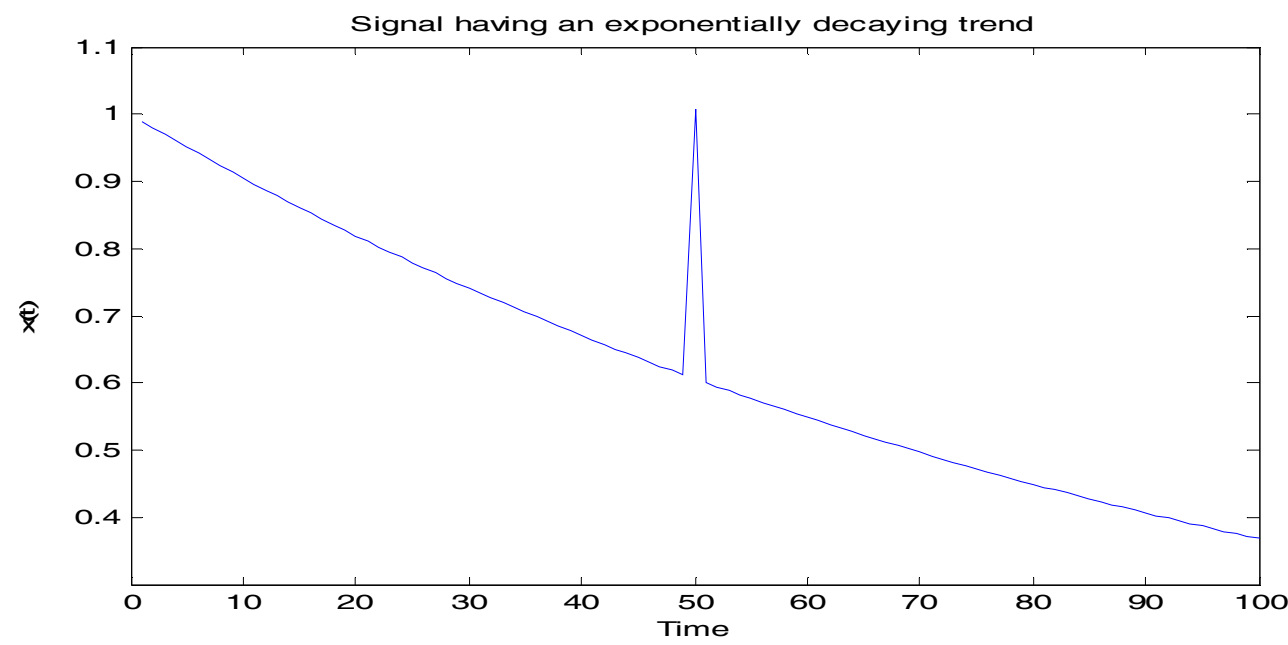

Figure 17: A pulse overlaid on an exponentially decaying trend signal

Separation of the pulse event after detrending using the CEEMD leads to excellent results as could be realized from figures 22 compared to thresholding of the original signal as shown in figure 18 . 


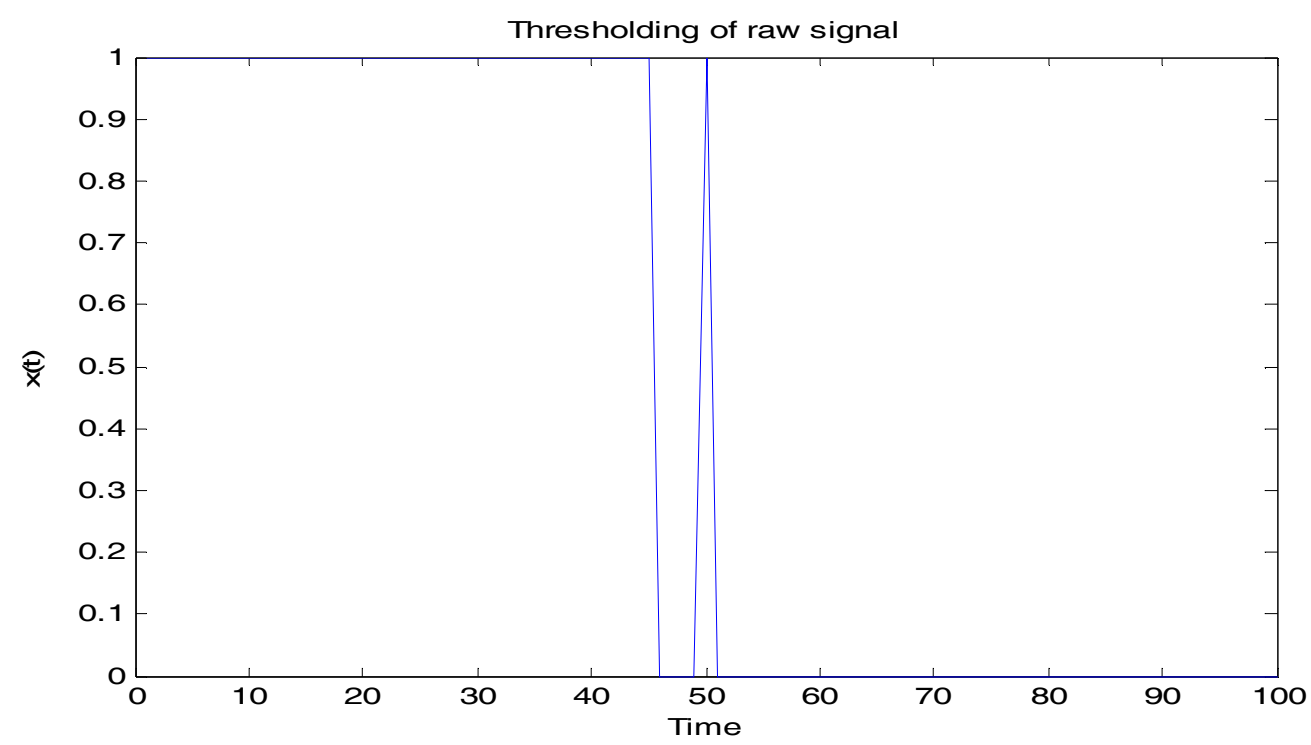

Figure 18: Thresholding using the raw signal based on $3.5^{*}$ standard deviation

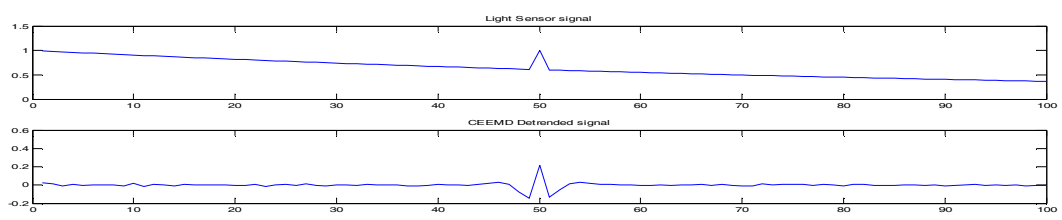

Figure 19: CEEMD Detrended Signal
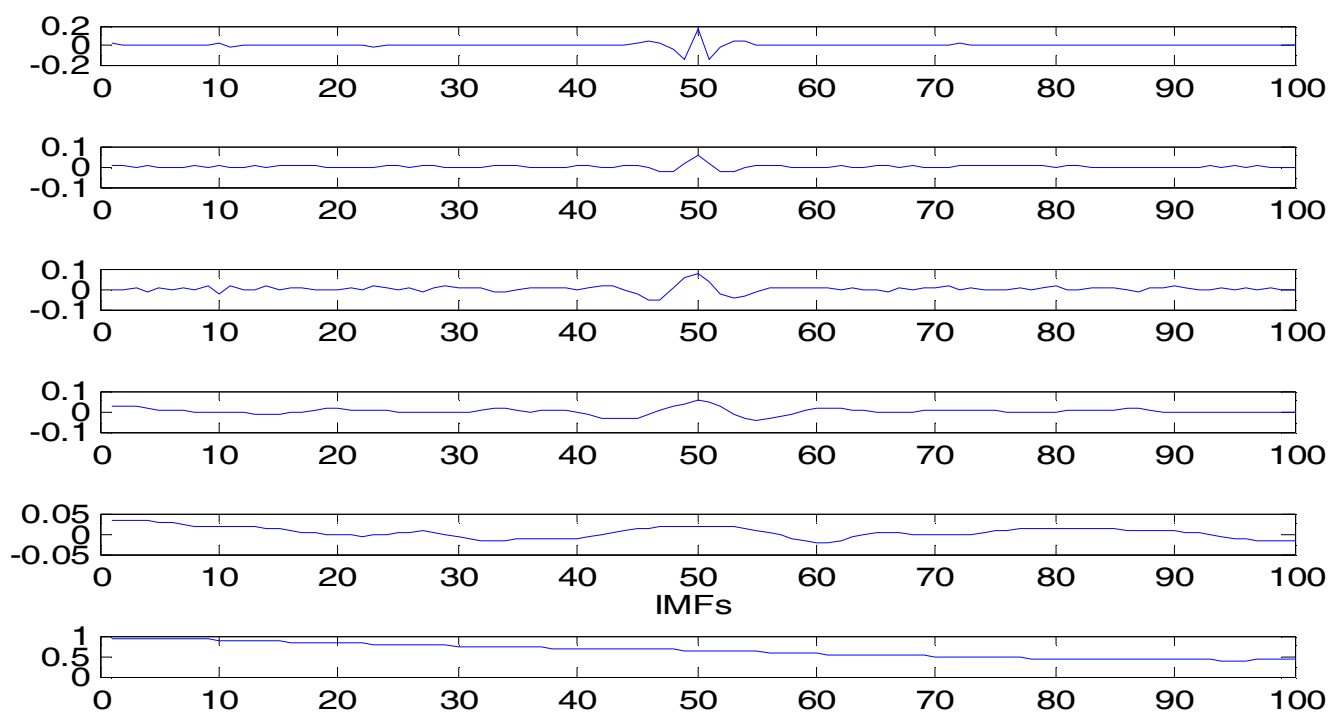

Figure 20: IMFs of the original signal 

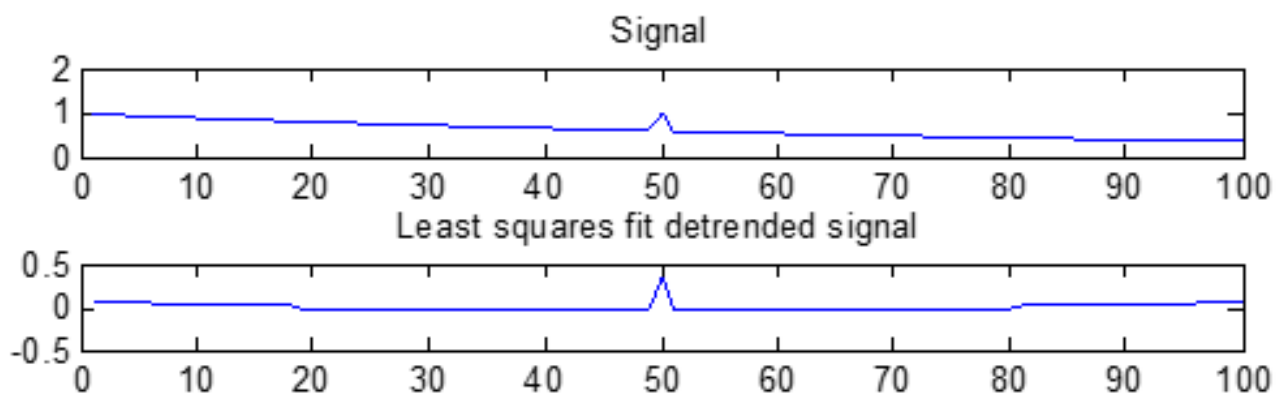

Figure 21: LSF Detrended Signal

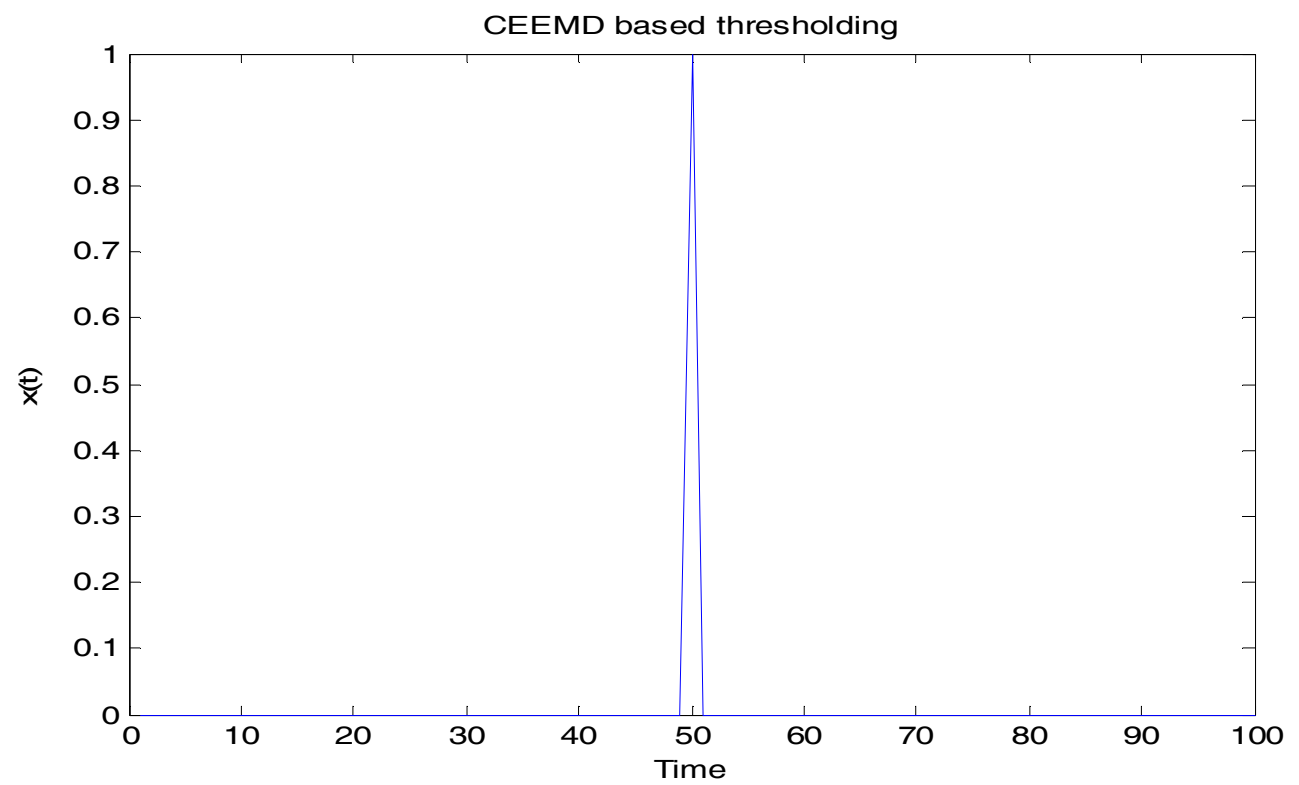

Figure 22: Thresholded Signal after trend removal using CEEMD

\section{Conclusions}

The Complete Ensemble Empirical Mode Decompositiom (CEEMD) is considered a means of nonstationary and non-linear signals analysis. This paper discusses the application of CEEMD to real-time trend removal in light signals. The rationale is that (CEEMD) method completely depends on the data that solves the mixed mode problem of EMD. The trend is described through a set of low-frequency Intrinsic Mode Functions (IMFs). The CEEMD trend removal was shown to perform well. The test results of both CEEMD based detrending and the well known least squares fit method indicate the superiority of the CEEMD detrending method. Statistical features extracted from the autocorrelation function have been used effectively to detect abnormal events using an LVQ classifier.

\section{Acknowledgements}

The authors would like to thank Torres ME, Colominas MA, Schlotthauer G, Flandrin P. for making the code of CEEMD available to the authors. 


\section{References}

1- AhmetMert and Aydin Akan, "DETRENDED FLUCTUATION ANALYSIS FOR EMPIRICAL MODE DECOMPOSITION BASED DENOISING,Proceedings of Eusipco 2014.

2- Arduino Uno. 2013. 2R Hardware \& Electronics: Arduino Uno. [ONLINE] Available at: http://2rhe.blogspot.fi/2011/04/arduino-uno.html. [Accessed April 2015].

3- Banzi, M. (September 2011). Getting Started With Arduino, 2.nd Ed, U.S.A., O'Reilly Books.

4- Blum, J. (2013). Exploring Arduino Tools and Techniques for Engineering Wizardry, Indiana, J. Wiley \& Sons.

5- Chengwei Li, Liwei Zhan and Liqun She, Friction Signal Denoising Using Complete Ensemble EMD with Adaptive Noise and Mutual Information, Entropy 2015, 17, 5965-5979

6- GiampieroCampa $\mathrm{PhD}$ "Learning Basic Mechatronics concepts using the Arduino Board and MATLAB", The MathWorks.

7- Http://Www.Arduino.Cc. [Accessed May 2014];

8- http://www.mathworks.com/matlabcentral/fileexchange/53172-denoising-signals-usingmorphological-techniques/content/Morphological\%20signal\%20filtering/morphological.m]

9- Huang, N.E. and Shen, S.S.P. "Hilbert-Huang Transform and Its Applications",World Scientific,(2005).

10- Huang, N.E., Shen, Z., Long, S.R., Wu, M.C., Shih, H.H., Zheng, Q., Yen, N., Tung, C.C., and Liu, H.H.: "The empirical mode decomposition and the Hilbert spectrum for nonlinear and nonstationary time series analysis". Proceedings of the Royal Society London A. 454,pp. 903-995,1998.

11- Huang, S., Http://Arduinodev.Com/Codeblocks.

12- LaureneFausett, "Fundamentals of Neural Networks: Architectures, Algorithms, and. Applications", Prentice-Hall, 1994.

13-MATLAB Support Package for Arduino Hardware: Read, write, and analyze data from Arduinosensors . Online Available at:

http://www.mathworks.com/hardware-support/arduino-matlab.html

14- Orfanidis, S.J., Optimum Signal Processing. An Introduction. 2nd Edition, Prentice-Hall, Englewood Cliffs, NJ, 1996.

15- Peter Waher, " Learning the Internet of Things", Packt Publishing, 2015.

16- S.A.Taouli\& F. Bereksi-Reguig,"ECG Signal Denoising by Morphological Top-Hat Transform" Global Journal of Computer Science and Technology Software \& Data Engineering Volume 13 Issue 5 Version 1.0 Year 2013.

17- Torres, M.E.; Lab. de Senales y Dinamicas no Lineales, Univ. Nac. de Entre Rios, Argentina ; Colominas, M.A.; Schlotthauer, G.; Flandrin, P. , A complete ensemble empirical mode decomposition with adaptive noise in IEEE International Conference on Acoustics, Speech and Signal Processing (ICASSP), 2011, p 4144-4147.] 